\title{
Positionnement descriptif, positionnement
} normatif, positionnement militant

Descriptive Positioning, Normative Positioning, Militant Positioning

\section{Marianne Doury}

\section{OpenEdition}

1 Journals

Édition électronique

URL : http://journals.openedition.org/aad/1540

DOI : 10.4000/aad.1540

ISSN : $1565-8961$

Éditeur

Université de Tel-Aviv

Référence électronique

Marianne Doury, « Positionnement descriptif, positionnement normatif, positionnement militant », Argumentation et Analyse du Discours [En ligne], 11 | 2013, mis en ligne le 15 octobre 2013, consulté le 23 septembre 2019. URL : http://journals.openedition.org/aad/1540 ; DOI : 10.4000/aad.1540

Ce document a été généré automatiquement le 23 septembre 2019.

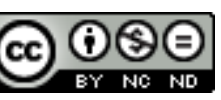

Argumentation \& analyse du discours est mis à disposition selon les termes de la licence Creative Commons Attribution - Pas d'Utilisation Commerciale - Pas de Modification 4.0 International. 


\title{
Positionnement descriptif, positionnement normatif, positionnement militant
}

\author{
Descriptive Positioning, Normative Positioning, Militant Positioning
}

\author{
Marianne Doury
}

1 La question du positionnement du chercheur vis-à-vis de son objet, de sa revendication d'une forme d'extériorité ou, à l'inverse, de «consubstantialité » à son égard, de la prise en charge (ou non) d'une dimension critique, traverse centralement les sciences sociales - au nombre desquelles on peut compter les études en argumentation, dès lors qu'elles se donnent comme objectif de contribuer à rendre compte d'échanges argumentatifs authentiques, qui constituent des objets sociaux, quel que soit le sens que prenne l'expression « objet social » selon le contexte d'enquête.

2 Cette question est si centrale, et a fait l'objet de tant de débats, en particulier dans le champ de la sociologie, qu'il est difficile de l'aborder de front et de chercher à l'organiser conceptuellement sur la base d'un «état de l'art » qui reprendrait les termes dans lesquels elle a été posée jusqu'à présent - même en restreignant le champ de la réflexion à un domaine disciplinaire : les études en argumentation ${ }^{1}$.

3 Aussi cet article cherche-t-il avant tout à rendre compte d'un parcours personnel en reprenant le fil d'une réflexion amorcée il y a près de dix ans dans un article intitulé «La position du chercheur en argumentation» (Doury 2004), et à en prolonger la posture, par un retour sur la façon dont la question de la dimension critique de l'analyse s'est posée pour moi au fil des années, les éléments de réponse que j'ai tenté d'y apporter, les zones de perplexité qui n'ont fait que gagner en ampleur, au point que la présente contribution s'oriente surtout vers la mise à plat de mes incohérences et de mon incapacité à les résoudre. Il s'agira de retracer la façon dont mon premier objet d'étude (le débat sur les parasciences) a été décisif dans le choix d'une approche purement descriptive (contre une perspective normative) de l'argumentation; et comment cette opposition entre approche descriptive et approche normative a été, dans une certaine mesure, brouillée, par des tentations militantes. En conclusion, une 
description sera menée en parallèle sur une étude épistémique et sur une étude militante de deux fragments d'argumentation politique, afin de montrer comment les deux registres sont marqués discursivement.

4 Le choix d'aborder le thème du numéro en le rapportant à une démarche personnelle ne doit être vu comme l'indice ni d'un narcissisme surdimensionné, ni d'une propension quasi-masochiste à battre ma coulpe : il repose sur l'idée qu'il y a de fortes chances que les questionnements qui se sont imposés à moi fassent écho à des interrogations rencontrées par plus d'un dans le domaine des études en argumentation; au moins, le caractère parfois très terre-à-terre des observations qui seront avancées ici donne-t-il des chances à cet article de faire autre chose que répéter, dans des termes nécessairement plus approximatifs, les grandes lignes du débat.

\section{Perspective normative / perspective descriptive}

5 Dans Doury (2004), j'ai tenté de retracer la façon dont, lors de mon travail de doctorat portant sur l'analyse de l'argumentation dans le débat sur les parasciences (parapsychologie, astrologie, ovnis, médecines parallèles, etc.), j'ai été immédiatement confrontée à des questions touchant à la dimension critique de l'analyse argumentative, et au choix entre une approche normative ou une approche descriptive de l'argumentation. Il s'agissait avant tout de faire face à des décisions concrètes, et en particulier : dans quelle section (références du corpus? références bibliographiques?) faire apparaître certains titres produits par des auteurs participant au débat analysé, mais élaborant des analyses critiques de ce débat très proches de celles qu'auraient pu en proposer des spécialistes d'argumentation d'inspiration normative. Je pense en particulier à l'ouvrage d'Alain Cuniot, Incroyable mais faux, ou aux deux livres d'Henri Broch, Au coeur de l'extra-ordinaire et Le Paranormal - ses documents, ses hommes, ses méthodes. Ces ouvrages visaient centralement à s'opposer au discours des défenseurs de diverses parasciences, ou plus spécifiquement, du "paranormal », sur la base d'une critique souvent serrée et parfois outillée des argumentations mises en œuvre².

Le choix entre perspective normative et perspective descriptive suppose d'opter pour un modèle théorique de l'argumentation (ou une famille de modèles), une méthodologie et des catégories d'analyse associées. Il détermine des questions de recherche qui structurent l'approche de l'argumentation de façon spécifique. A gros traits, dans une perspective normative, l'analyste cherche à répondre à la question: "cet argument est-il ou non un bon argument?", alors que l'analyste descriptif cherche avant tout à répondre à la question «comment cette argumentation fonctionne-t-elle? » (« comment ça marche?»).

7 Ma réflexion a été guidée par l'observation que, lorsqu'il choisit une approche théorique normative de l'argumentation, et qu'il adopte des catégories d'analyse visant à évaluer l'argumentation, ce que produit l'analyste de l'argumentation ne diffère pas, par nature, des contributions des participants au débat qu'il analyse - autrement dit, la position de l'analyste est largement superposable à celle des acteurs. On peut montrer en effet que les systèmes évaluatifs qui sous-tendent les modèles normatifs savants de l'argumentation ne diffèrent pas fondamentalement des principes normatifs auxquels adhèrent les locuteurs ordinaires engagés dans des discussions argumentatives. Par exemple, dans une recherche antérieure (Doury 2008), j'ai tracé les expressions «ce n'est pas un argument » et « ça n'a pas valeur d'argument », critiques adressées par des 
internautes en réaction à des messages postés sur des forums de discussion. J'ai prêté attention à ce qui constituait la cible de telles critiques, et j'ai analysé la façon dont de telles condamnations ( "ce n'est pas un argument », «ça n'a pas valeur d'argument »), condamnations fortes puisqu'elles ont pour effet, littéralement, de rejeter les discours produits hors du champ de l'argumentation, étaient justifiées par leurs auteurs.

Voici quelques occurrences de ces critiques :

«Tout le monde le fait» n'est pas un argument, l'erreur commune n'est pas une vérité ${ }^{3}$.

citer sarkozy a chacune de tes interventions se n'est pas un argument... un argument c'est répondre a une chose par des faits concrets qui se sont passé $e^{4}$ !

AMHA $^{5}$, l'absence d'argument contre une idée n'est pas un argument significatif pour valoriser l'idée ; ${ }^{6}$

il n'empêche qu'une chanson n'est pas un argument ${ }^{7}$

Chez eux la sympathie se mérite et le coup de trique n'a pas valeur d'argument ${ }^{8}$.

Le vécu, et l'expérience personnelle n'ont pas valeur d'arguments car ils ne sont pas universels et ne permettent donc pas de faire avancer le débat ${ }^{9}$.

L'étude systématique de commentaires méta-argumentatifs de ce type met au jour une " argumentologie populaire », comme on parle de "linguistique populaire », c'est-àdire quelque chose comme une proto-théorisation spontanée de l'argumentation, qui voit celle-ci comme une activité rationnelle, basée sur le rejet des émotions, et conçue comme une alternative à la contrainte physique, à la force. Selon cette conception de l'argumentation, les arguments doivent avoir une portée générale; un raisonnement qui apparaîtrait trop spécifique (comme les arguments inductifs du dernier exemple cité) court le risque de se voir dénier "toute valeur d'argument ». Il en est de même lorsqu'un argument est indissociable de la personne qui l'avance ; c'est le cas à chaque fois qu'on fait dépendre l'évaluation d'une proposition de l'évaluation de la personne qui la soutient. L'évaluation peut être positive (c'est le cas de l'argument d'autorité), elle peut être négative (c'est le cas de la disqualification ad hominem). Quoi qu'il en soit, tout discours faisant dépendre l'évaluation d'une proposition de l'évaluation de la personne qui la porte a de fortes chances, en contexte polémique, de se voir refuser le statut d'argument.

En bref, les types d'arguments stigmatisés comme déviants par les locuteurs ordinaires appartiennent souvent au répertoire des fallacies (paralogismes, sophismes) identifiées au fil des siècles par les théoriciens de la rhétorique, de l'argumentation et de la logique (ad hominem, ad verecundiam, ad populum), ou à des raisonnements souvent pointés comme particulièrement fragiles d'un point de vue logique par les analystes (argument par analogie, argument inductif...). La similarité entre les normes mobilisées dans les deux contextes est si forte qu'il devient difficile de poser l'existence d'une différence de nature entre l'activité de contre-argumentation adossée à une critique "méta » de l'argumentation adverse prise en charge par les participants à une discussion argumentative, et l'activité d'évaluation de l'argumentation menée par l'analyste adoptant une perspective normative savante.

11 Il est possible d'assumer cette proximité, et d'accepter que la position de l'analyste s'inscrive dans un continuum avec la critique ordinaire de l'argumentation, dont elle ne différerait pas par nature. Certains auteurs tenants d'une approche normative ont ainsi souligné les liens entre contre-argumentation, évaluation «savante» et évaluation 
« spontanée » des arguments (voir notamment van Eemeren et Meuffels 2002, Garssen 2002 ; Govier 1987, 2001). Il s'agit alors, pour l'analyste normatif « savant »,

- d'expliciter et de systématiser le recours à des normes argumentatives ;

- de prendre garde à ce que l'évaluation de l'argumentation ne dérive pas vers un engagement ${ }^{10}$ militant qui se donnerait comme une analyse normative à visée épistémique.

12 Pour ma part, j'ai cherché à maximiser la distance entre ma position d'analyste et l'activité argumentative produite par les locuteurs dans les données analysées; j'ai donc opté pour une perspective purement descriptive sur l'argumentation.

Un tel choix ne conduit pas à revendiquer une rupture radicale et totale entre l'activité du locuteur à l'origine des données étudiées, et l'activité de l'analyste «savant " de l'argumentation; d'abord parce que tous deux sont soumis à l'humaine condition de locuteurs-argumentateurs, à sa part inhérente de subjectivité, au surgissement d'enjeux qui traversent nécessairement leur parole. Mais le choix d'une perspective descriptive permet à l'analyste de ne pas partager le même « objet du débat " (Sitri 2003) que le locuteur dont il étudie les propos. Si on peut considérer l'analyse qu'il produit comme une prise de position, du moins ne porte-t-elle pas sur la même question que celle sur laquelle les débatteurs se positionnent; c'est cette capacité de déplacement, pour reprendre les termes de Heinich (2002: 124), qui constitue ce que l'analyste de l'argumentation peut apporter en propre. En particulier, les locuteurs engagés dans un débat, au-delà de l'activité d'étayage argumentatif de la position qu'ils défendent, se livrent à une intense activité de critique de l'argumentation adverse critique largement soumise à un objectif stratégique de disqualification. En optant pour une approche descriptive de l'argumentation, l'analyste s'autorise à ne pas partager ce questionnement critique, et à ne pas doubler l'évaluation produite par les locuteurs dans les données analysées par sa propre évaluation.

Ce choix ne conduit pas pour autant à négliger la question des normes en argumentation: elles sont une composante importante de ce que l'analyste de l'argumentation doit décrire. Une partie de son entreprise consiste à rendre compte de la façon dont les locuteurs ordinaires manifestent leur adhésion à des normes argumentatives, par exemple lorsqu'ils rejettent l'argumentation adverse au motif qu'il s'agirait d'un "amalgame ", d'un " procès d'intention ", d'un " argument d'autorité ", etc. ${ }^{11}$ : «a major part of any description will be a reconstruction of people's own normative ideas. That is, in order to adequately describe argumentative practice, we must realize that people already have ideas about whether and how they are obliged to defend their statements » (Jackson, 1989: 113).

Cette position étant explicitée, je souhaiterais mentionner deux réactions auxquelles je suis confrontée régulièrement dès lors que je revendique une approche descriptive de l'argumentation.

La première a une tonalité psychologisante, et on m'accordera, je pense, ce qu'elle peut avoir d'irritant. Elle connaît différentes variantes, qui toutes reviennent à la question suivante : «mais de quoi as-tu peur?»; le refus de partager les mêmes questions que les locuteurs dont j'analyse les propos (et en particulier, celles touchant à l'évaluation des arguments des débatteurs) étant interprété comme une forme de frilosité du chercheur retranché dans sa tour d'ivoire, une volonté quasi-névrotique de "rester propre ", de préserver la "pureté » de l'analyse du caractère nécessairement «crapoteux » de l'engagement argumentatif. Le "courage» serait alors du côté des 
défenseurs d'une position normative, voire d'une perspective engagée, en argumentation. Cette caractérisation des positions va de pair avec une perception de la première position comme dominante dans le champ des études en argumentation, et de la seconde, comme minoritaire. Il me semble pour ma part que dès lors que l'on prend en considération les études de l'argumentation qui se développent dans le domaine anglo-saxon, les proportions sont clairement inversées (en raison notamment de l'influence de la logique informelle dans cette sphère); la revendication, par certains tenants d'une approche critique de l'argumentation, d'une position minoritaire, dissidente et « courageuse » me semble relever d'une stratégie rhétorique plus que de la description étayée d'un champ scientifique (voir les remarques similaires dans Heinich, 2004 : 159).

La seconde réaction suscitée par une prise de position descriptive en argumentation revient cette fois non pas à pointer une forme de défaillance psychologique, mais à instaurer quelque chose comme une hiérarchie conceptuelle et méthodologique entre description et évaluation. "D'accord», dit-on, «le travail de description que vous proposez est utile ; c'est un préalable nécessaire à l'entreprise normative qui, sur la base de l'identification des procédés argumentatifs utilisés, va les évaluer et dire ce qu'ils devraient être par rapport à un idéal argumentatif ». Dans cette perspective, la description de l'argumentation ne serait pas un objectif en soi, mais une sous-tâche de l'analyse argumentative, subordonnée à un objectif supérieur d'évaluation et de préconisation. Je serais prête à assumer ce cadrage de mon choix théorique et méthodologique, et à accepter ce statut de " petite main » (ne voyant rien de dégradant dans l'approche empirique) s'il n'apparaissait clairement que le travail de description va de pair avec la construction d'un modèle de l'argumentation, dans un va-et-vient incessant entre empirie et théorisation, et que les modèles en adéquation avec une approche descriptive de l'argumentation ne sont pas nécessairement les mêmes que les modèles au service d'une entreprise normative. Par exemple, la proposition de reconstruire les échanges argumentatifs selon le modèle de la discussion critique, qui distribue les interventions en quatre étapes (confrontation, ouverture, argumentation, conclusion), est au cœur du modèle pragma-dialectique, et ne se justifie que par l'objectif évaluatif qu'il se donne (certains « coups » argumentatifs étant attendus à une étape donnée, et fallacieux à d'autres moments). En revanche, une approche descriptive de l'interaction argumentative, visant notamment à rendre compte de l'émergence et de l'évolution des stases et des mécanismes interactionnels de coalition, s'adossera à un modèle refusant de briser, par l'analyse, la linéarité des échanges.

Au-delà de ces premiers commentaires, les deux réactions (descriptivisme comme symptôme de frilosité, descriptivisme comme préalable à l'approche évaluative) appellent d'une certaine façon une réponse commune, qui revient à spécifier mon champ de compétence, et à revendiquer le droit à déterminer les questions qui m'intéressent. Mon approche de l'argumentation est caractérisée par un ancrage dans le champ des sciences du langage. C'est à travers leur inscription langagière, leur contribution à la cohérence textuelle, les dynamiques interactionnelles qui les contraignent et qu'ils orientent en retour, que je peux saisir les mécanismes argumentatifs. C'est parce que les normes argumentatives à l'œuvre dans les échanges sont saisissables à ces trois niveaux - inscription langagière, cohérence textuelle, dynamique interactionnelle - que j'ai quelque chose à en dire; mais je ne vois pas quelle légitimité j'aurais, en tant que linguiste (ou analyste du discours, ou représentante d'une approche langagière de l'argumentation), à redoubler l'activité 
évaluative des locuteurs dont j'étudie les propos en produisant, justifiant et appliquant mon propre système de normes argumentatives. Je ne dis en aucun cas que les approches normatives de l'argumentation sont absurdes, ou démesurément ambitieuses, ou illégitimes ${ }^{12}$ : elles s'attachent en revanche à répondre à des questions que je considère comme hors de mon champ d'investigation dès lors que je m'attache aux manifestations langagières de l'argumentation; et ce positionnement me paraît incontestable, à condition de ne pas prétendre épuiser l'objet par cette approche.

\section{Une distinction brouillée dans la pratique}

Voilà mes orientations théoriques et méthodologiques clairement posées. Malheureusement, leur mise en œuvre, dans la diversité de l'exercice de mes pratiques professionnelles, est loin d'avoir la belle cohérence qu'on pourrait souhaiter, et je suis, comme beaucoup, tiraillée entre valeur de détachement et valeur d'engagement (Heinich, $2002: 122$ ).

D'abord, parce que si, sur le principe, l'analyste n'a à se positionner ni sur l'objet du débat sur lequel il travaille, ni sur l'évaluation des argumentations que les locuteurs y déroulent, en réalité, "les préférences de l'analyste restent souvent accessibles à travers son analyse, ce qui montre bien que chercher à faire en sorte que l'analyste « ne soit plus présent dans son texte » est un objectif dans une certaine mesure inaccessible - et de toute façon, sans doute peu souhaitable» (Doury 2004:151). C'est la conséquence du fait que, comme le souligne Koren (2003), le chercheur n'échappe pas au régime général de l'énonciation, qui veut que la façon même dont il s'inscrit dans (ou se désinscrit de) son discours fait sens, dit des choses de lui, de son positionnement dans l'espace socio-discursif, de ses préférences ou répulsions par rapport à son objet de discours.

Il me semble qu'il faut apporter deux réserves quant aux conséquences que l'on pourrait être tenté de tirer de cette affirmation. D'une part, s'il est indéniable que, en réception, le destinataire d'un discours tire des inférences, à partir des éléments éthotiques mis à sa disposition, sur celui qui en est à l'origine, ces inférences se font sur la base d'une construction sémiotique dont rien ne dit qu'elle correspond à la réalité du locuteur comme personne - soit qu'il se soit livré délibérément à une " mise en scène " de lui-même contre-factuelle (que cette mise en scène soit mensongère, ou simplement ludique ou esthétique), soit que l'image qu'il renvoie de lui lui ait échappé ; je pense ici à la surprise que j'ai ressentie lorsque, à l'occasion de ma soutenance de thèse, j'ai découvert qu'un membre de mon jury, que j'avais lu et relu, et dont le prénom ne laissait rien présager de son genre, était un homme, alors que je m'en étais forgé une image féminine sur la base de ses écrits (ce dont j'ai d'ailleurs pris conscience dans le même temps que cette image était contredite par les faits).

Par ailleurs, que l'analyste soit traversé par des préférences diverses, et que celles-ci soient susceptibles de transparaitre dans ses discours en général, et dans ses analyses en particulier, n'implique en rien que sur le fond, la méthodologie qu'il adopte et les résultats auxquels il aboutit seraient radicalement différents si sa position sur le sujet débattu avait été tout autre - sans quoi autant renoncer tout de suite à la prétention à une forme de scientificité.

Plus généralement, on peut recourir une fois encore à Heinich (2002:120, $2004: 153)$, et à la distinction qu'elle propose entre valeurs au sens large (manifestations implicites 
d'une position dans l'espace social) et valeurs au sens restreint (préférences communiquées intentionnellement); mais dans les faits, il n'est pas toujours facile de délimiter clairement la frontière qui sépare la nécessaire inscription de tout locuteur (chercheur compris) dans ses productions discursives (articles scientifiques compris), et une prise de position assumée sur l'objet d'analyse. Ainsi, si, dans la production de mes textes écrits, j'arrive dans l'ensemble à tenir le positionnement descriptif posé précédemment, lors de mes interventions orales (tant en colloque qu'en séminaire), on m'a fait remarquer le ton ironique, voire persifleur, qu'il m'arrivait d'employer pour commenter certaines argumentations particulièrement goûteuses, et le vocabulaire indéniablement évaluatif que je mobilisais alors. Je devrais pouvoir contrôler cette irruption du locuteur ordinaire que je suis aussi au cœur de mon activité académique ; mais je ne suis pas sûre d'en avoir la volonté, et je tends à m'autoriser, jusqu'à ce que j'en décide autrement, à ménager, au cœur de ma pratique experte, quelques espaces d'expression quasi-ludique de mes inclinations naturelles.

Par ailleurs, force est de constater que, si on élargit un peu la focale, il existe une pression assez forte, tant externe qu'interne, pour un glissement vers un cadre évaluatif, voire vers une perspective militante. Cette pression, d'un positionnement de chercheur à un positionnement de penseur, dans les termes de Heinich (2002), se fait sentir particulièrement dès lors que s'esquisse la possibilité, pour l'analyste "savant ", de s'adresser à un public un peu plus large que ses pairs. En particulier, invité par les médias à réagir, par exemple, sur un débat télévisé, les questions qu'on lui pose sont généralement de l'ordre de " qui a raison? qui a tort ? qui a gagné, qui a perdu ? ${ }^{13}$, ce qui suppose une hiérarchisation des arguments en termes de vérité / fausseté ou d'efficacité / inefficacité, et donc une perspective évaluative. Plus généralement, dans l'article qu'elle consacre à la sollicitation récurrente, par les médias, d'« expertssociologues", Aurélie Tavernier (2012) insiste sur le fait que ces derniers sont constamment invités à édicter des normes, et à basculer ainsi, «du registre de la description à celui de la prescription "; elle cite notamment le sociologue Jean-Claude Kauffman, qui, sur la base de sa propre expérience des médias, affirme : «Il y a une attente de prescription de normes [...], de toute façon on va toujours me demander “mais qu'est-ce qu'il faut faire" » (2012:41).

Par ailleurs, la tentation d'un engagement militant, cette fois, peut être le fait de l'analyste lui-même, tenté de toucher un public plus vaste et de conférer une signification sociale à une activité scientifique qui lui semble par moments excessivement désincarnée et détachée de tout enjeu « réel ». Cette tentation peut le conduire à prendre en charge, momentanément ou durablement, une visée militante et à prétendre, par une analyse experte de l'argumentation, contribuer à "changer le monde $»^{14}$.

Il convient à ce point du raisonnement de préciser que l'adoption d'un cadre d'analyse normatif en argumentation ne doit pas se confondre avec l'adoption d'une perspective militante. C'est là où les approches évaluatives savantes se distinguent de droit des pratiques évaluatives ordinaires de l'argumentation. Si les locuteurs engagés dans des échanges argumentatifs, comme les scientifiques qui les analysent, cherchent à évaluer les arguments auxquels ils sont confrontés à l'aune de critères plus ou moins explicites déterminant ce qui relève de la «bonne » ou de la «mauvaise» argumentation, les premiers mettent le plus souvent cette évaluation au service d'objectifs stratégiques de réfutation du discours de l'adversaire, ou de soutien aux discours alliés. Pour les 
analystes savants, en revanche, le travail s'achève avec l'évaluation, qui constitue une fin en soi, et n'est au service d'aucune position dans le débat. En principe du moins; il faut reconnaître que souvent, dans les travaux relevant d'approches normatives de l'argumentation, les exemples visant à illustrer des paralogismes sont empruntés à des discours défendant des positions relevant d'un pittoresque farfelu (discours empruntés aux partisans de diverses pseudo-sciences dans les manuels de critical thinking nordaméricains) ou d'idéologies infâmantes (discours antisémite, discours d'extrême-droite dans les travaux de Breton ou de Koren). Comme le souligne Plantin (1998), « Si l'on se propose de construire une théorie critique de l'argumentation, ayant pour objectif de traquer sophismes et paralogismes, on sera tenté de prendre plutôt pour objet des textes que la communauté réprouve ». La disqualification des procédés fait alors écho à la disqualification des thèses défendues, au point qu'analyse normative et engagement militant deviennent parfois difficiles à distinguer ${ }^{15}$. Symétriquement, la subordination d'analyses argumentatives à des fins militantes passe souvent par la mobilisation d'un arsenal d'analyse normatif, ce qui permet de conférer une forme de caution savante à un positionnement avant tout politique.

\section{Approche contrastive de deux types d'analyses argumentatives}

Je voudrais poursuivre ma réflexion en évoquant ma propre expérience d'analyste du discours politique. Cette expérience a suivi deux voies différentes. La première s'inscrit dans un positionnement descriptif épistémique et visant à une neutralité axiologique : elle prétend essentiellement, à travers l'analyse argumentative de données, à accroître la connaissance, aussi bien sur l'objet étudié que sur les mécanismes argumentatifs mis au jour et, en retour, sur la théorie de l'argumentation. Je l'illustrerai par un travail que j'ai mené récemment sur l'analyse du débat de l'entre-deux tours qui a opposé François Bayrou à Ségolène Royal lors des élections présidentielles françaises de 2007.

Le deuxième type d'analyse que je voudrais évoquer est celui auquel je me suis livrée à deux reprises en 2011 à propos de discours politiques qu'il s'agissait, pour moi, d'analyser afin de les dénoncer comme politiquement défaillants. Une de ces études a porté sur des propos tenus par Claude Guéant, alors ministre de l'Intérieur, propos qu'il s'agissait de dénoncer comme discriminatoires; l'autre a cherché à mettre au jour une "rhétorique de l'évidence" dans le discours de Nicolas Sarkozy, rhétorique qu'il s'agissait de dénoncer comme à la fois mensongère et dangereuse.

29 Je vais à présent rendre compte brièvement de ces deux types de recherche. Je présenterai dans un premier temps l'étude $\mathrm{E}$ (pour Epistémique), sur le débat BayrouRoyal; puis je présenterai l'étude $M$ (pour Militant), sur les déclarations de Claude Guéant. Je discuterai enfin quelques caractéristiques propres à chaque approche.

\section{Etude E}

L'étude E porte sur la fonction de l'accord dans l'argumentation polémique - et plus particulièrement, dans le débat qui a opposé François Bayrou et Ségolène Royal après le premier tour des élections présidentielles françaises de 2007, scrutin au terme duquel les deux finalistes (Nicolas Sarkozy avec 31,1\% et Ségolène Royal, avec 25,8 \%) sont 
suivis par François Bayrou, représentant du Modem, qui réalise le score surprenant de $18,5 \%$ et se retrouve donc en position d'arbitrer le second tour.

Cette recherche part d'une observation quelque peu paradoxale :

- Les théories de l'argumentation posent qu'argumenter suppose l'existence d'un désaccord sur une question donnée ; ce désaccord peut être exprimé explicitement par les participants au débat, ou doit au moins pouvoir leur être attribué raisonnablement. - Mais par ailleurs, les théories de la politesse considèrent que le désaccord, lorsqu'il surgit, signale l'existence d'un problème interactionnel.

Ainsi, en contexte argumentatif, l'émergence d'un désaccord est à la fois probable voire nécessaire, et problématique (en ce qu'elle amène avec elle des problèmes de gestion des faces au sens de Goffman). Aussi peut-on s'attendre, dans de tels contextes, à observer la mobilisation de procédés discursifs et interactionnels visant à atténuer l'expression des désaccords, et à accentuer l'expression des accords ${ }^{16}$.

Pourtant, l'analyse de l'expression des accords et des désaccords lors du débat BayrouRoyal montre que le principe de préférence pour l'accord est largement déterminé par des considérations propres à la situation d'énonciation : par les contraintes inhérentes au genre «débat télévisé " (et même, plus précisément, "débat de l'entre-deux tours »), et par les contraintes liées aux objectifs politiques propres aux participants. Durant ce débat, François Bayrou met essentiellement en œuvre une stratégie de différenciation, et insiste ainsi sur les divergences, alors qu'il atténue les points d'accord avec son interlocutrice. Cette stratégie est liée à ses objectifs politiques: il cherche à substituer à l'opposition binaire droite/gauche qui gouverne traditionnellement la vie politique française un "ménage à trois", au sein duquel il représenterait le troisième larron (le parti centriste). Le registre qu'il privilégie au cours du débat est donc le désaccord renforcé, tant vis-à-vis de Nicolas Sarkozy (absent) que de Ségolène Royal, qui constitue sa partenaire d'interaction :

FB : non (.) non (.) non (.) non pas du tout (.) pas du tout ça n'est pas le pays qui paye ça n'est pas l'endettement du pays ça n'est pas l'Etat qui paye les retraites (.) vous avez une confusion (.) entre les deux (.)

34 L'expression frontale du désaccord («non ») est renforcée par la répétition multiple du «non ». Elle est suivie par l'expression du caractère radical du désaccord (" pas du tout »), lui-même répété. Suit l'utilisation d'une négation polémique qui reprend pour les contester les mots mêmes de Ségolène Royal ("ça n'est pas...»), pour ensuite justifier son désaccord par une caractérisation négative du raisonnement de son adversaire («vous avez une confusion entre les deux »).

Les éléments contextuels qu'on a évoqués orientent symétriquement S. Royal vers l'atténuation de l'expression des divergences et l'accentuation des points d'accord. Cette stratégie est conforme à son intérêt politique, qui est d'amener François Bayrou à appeler son électorat à voter pour elle contre Nicolas Sarkozy au second tour. Cette volonté de souligner les convergences l'incline à privilégier fortement les ententes, même lorsque l'orientation donnée par François Bayrou lors de son intervention précédente insiste sur les divergences. C'est ce qu'on peut observer par exemple dans l'enchaînement suivant (qui fait suite à une longue intervention au cours de laquelle François Bayrou critique fermement la politique qu'il juge excessivement étatique de $\mathrm{S}$. Royal) :

FB : c'est un des chapitres sur lesquels nous sommes en désaccord profond $\mathrm{J}$ : Ségolène Royal 
SR : [inspiration] d'abord je je constate: avec euh: satisfaction / que nous venons d'acter déjà des convergences sur la réforme des institutions/ sur l'Etat impartial/ sur une certaine vision (.) de l'Europe et en particulier sur le dernier point qui a été évoqué/ [suit une tentative de Ségolène Royal de montrer que sur un des points évoqués par F. Bayrou, il n'y a pas réellement désaccord]

Alors que François Bayrou, concluant de façon très nette sur l'existence de divergences de vue, invite sa partenaire d'interaction à enchaîner sur ce point, $S$. Royal préfère réagir d'abord à l'expression des quelques objets d'accord concédés par l'ancien candidat centriste précédemment, objets d'accord dont elle accentue l'importance par une construction énumérative.

Les stratégies divergentes adoptées par chacun des débatteurs permettent de restituer la logique qui sous-tend des échanges aussi baroques que le suivant, toujours suite à l'affirmation d'un désaccord de François Bayrou (cette fois sur le mode de financement de l'augmentation des retraites et bas salaires) :

$\mathrm{FB}$ : on est en désaccord sur ce point

SR : non non (.) non non non (rire) on n'est pas en désaccord sur ce point (.) je suis

(.) je suis au contraire très satisfaite que vous puissiez enfin reconnaître (.) qu'il y a des dépenses qui sont absolument NEcessaires/

La stratégie, récurrente chez Ségolène Royal, qui consiste à accentuer les accords jusqu'à la dénégation de désaccords pourtant assumés par son partenaire d'interaction, a ceci de surprenant qu'elle l'amène à se poser comme seule légitime à identifier les points d'accord ou les divergences irréductibles entre elle et son partenaire d'interaction, quoi qu'en dise ce dernier. D'une certaine façon, elle revient à afficher, dans le cours du débat, l'issue positive de la discussion, et à poser des relais qui lui permettront de conclure à la réussite de l'entreprise de rapprochement.

39 En résumé, la préférence pour l'accord ou le désaccord, dans ce débat, reflète et renforce les enjeux politiques et les objectifs poursuivis par chacun de ces acteurs-clés de l'élection présidentielle. Après avoir identifié les stratégies respectives des candidats au regard de cette préférence pour l'accord ou le désaccord, je me suis intéressée à la façon dont les accords étaient formulés; et j'ai montré que cette formulation ellemême jouait un rôle dans la négociation des rapports de force entre les deux débatteurs.

40 Il est ainsi apparu que la locution verbale "être d'accord avec", bien qu'étant un prédicat à deux arguments, n'est pas absolument symétrique ${ }^{17}$, et il est clair que pour les candidats, « $\mathrm{X}$ est d'accord avec $\mathrm{Y}$ » n'est en rien équivalent à " $\mathrm{Y}$ est d'accord avec $\mathrm{X}$ » : l'expression suggère que dans le premier cas, $\mathrm{X}$ « rejoint » $\mathrm{Y}$ sur une position qu'il occupait auparavant. $\mathrm{Y}$ occupe alors en quelque sorte une position haute, puisqu'il bénéficie de l'antériorité et de l'initiative sur le sujet en question; il fait figure, d'une certaine façon, de leader d'opinion. Dans un débat politique, celui qui dirait à son rival « je suis d'accord avec vous » donnerait ainsi le sentiment de lui faire allégeance.

41 Aussi les participants évitent-ils d'utiliser la phrase « je suis d'accord avec vous sur P ", et préfèrent recourir à des expressions du type "nous sommes d'accord sur $\mathrm{P}$ », qui mettent les deux partenaires sur un pied d'égalité. François Bayrou dit ainsi souvent " on a des accords", « on a des convergences ». En revanche, lorsqu'il s'agit d'affirmer sa spécificité et de se distinguer de son adversaire politique socialiste, François Bayrou utilise sans réticence le prédicat asymétrique « je suis en désaccord avec... ». En effet, alors que l'expression " je suis d'accord avec $\mathrm{X}$ " suggère, de la part du locuteur, un mouvement de ralliement avec X (et fait donc l'objet de la part des débatteurs de 
stratégies d'évitement), « je suis en désaccord avec $X$ » suggère que le locuteur entre en conflit, prend l'initiative de la rupture, et constitue donc une ressource discursive prisée.

De façon systématique, tout au long du débat, Bayrou et Royal privilégient les modalités d'expression de l'accord qui construisent un rapport de force qui leur est favorable, comme « ceci est vrai », «j'approuve cette idée », « cette idée est bonne » (expressions qui mettent le locuteur dans la position forte de celui qui évalue l'autre), ou même "c'était dans mon programme ", ce qui est une façon un peu infantile de revendiquer la paternité de la proposition objet d'accord.

Il arrive toutefois que l'expression «je suis d'accord avec vous » soit utilisée; son interprétation possible comme indice de ralliement est alors désamorcée par le recours à divers procédés, dont je présenterai deux exemples ici :

- L'accord porte sur une position que l'adversaire a défendue il y a longtemps, mais le désaccord l'emporte si l'on considère les positions défendues par les deux débatteurs au moment du débat.

Ainsi, à la demande d'un journaliste qui invite Bayrou à réagir au positionnement de Ségolène Royal sur les 35 heures, il répond :

FB : alors pour les points d'accord et de désaccord sur les $35 \mathrm{~h}$ il faudrait que je sache avec quelle Ségolène Royal je parle parce qu'il y a eu une Ségolène Royal avec qui j'étais profondément en accord c'est quand elle est entrée dans sa campagne interne (.) y a ici des experts (.) elle est entrée dans sa campagne interne (.) elle a dit les trente-cinq heures/ franchement/ c'est pas un exemple/ et euh ça s'est mal fait puis après elle a eu des difficultés avec euh l'intérieur du parti socialiste ce que je ce que j'comprends ça arrive/ (.) et euh et elle a été euh elle a f- elle a transformé son propos en (.) je veux une généralisation des trente-cinq heures (.) c'est une très mauvaise idée à mes yeux (.) c'est une très mauvaise idée parce que les entreprises qui vont être ainsi concernées par les 35 heures c'est les petites entreprises (.) les plus p'tites entreprises [...] et je suis en en désaccord sur ce point

Ponctuellement, la formule « je suis d'accord avec X» est acceptable pour Bayrou au début de son intervention dans la mesure où elle est associée à une désactualisation temporelle : elle valait pour l'« ancienne Ségolène ", celle de la campagne interne ; mais à la fin de l'extrait, Bayrou revient à la formule préférée d'expression des divergences, " je suis en désaccord », lorsqu'il s'agit de prendre position sur la Ségolène actuelle.

- Une seconde stratégie visant à neutraliser l'effet d'allégeance produit par l'expression « je suis d'accord avec vous » consiste à l'intégrer comme l'amorce d'une construction concessive : « je suis d'accord avec vous, mais »; c'est ce que fait Ségolène Royal, après que Bayrou a proposé d'augmenter les petites retraites :

SR : (1) [je suis d'accord (.) il faudrait donner plus (.) y compris le smic pour aller plus vite (.) y compris les petites retraites] (.) (2) [mais nous devrons le faire en tenant compte de l'endettement du pays]

L'expression de l'accord en (1) est immédiatement suivie d'un ajout à la proposition de Bayrou ("y compris le smic ») qui constitue une surenchère par rapport au discours initial à la Dupont et Dupond ( «je dirais même plus»). Cette stratégie permet à Ségolène Royal de reprendre l'initiative des propositions. Par ailleurs, (2) introduit une condition à la réalisation de (1), et est présenté comme l'essentiel du propos de Ségolène Royal, ce sur quoi elle invite à enchaîner, alors que l'objet d'accord, (1), est remisé à l'arrière-plan. 
47 L'étude E conclut sur le fait que la préférence structurelle pour l'accord, posée par les conversationnalistes, peut être suspendue pour au moins deux types de raisons :

- des contraintes génériques : certains genres interactionnels centrés sur le désaccord peuvent amener à suspendre, voire à renverser, la préférence pour l'accord ;

- des contraintes stratégiques : la préférence pour l'accord peut être suspendue dès lors qu'elle est susceptible de compromettre la réussite des objectifs d'un des débatteurs.

\section{Etude M}

Passons à présent à la présentation de l'analyse argumentative d'un discours politique que j'ai produite dans le cadre de l'Etude $\mathrm{M}$ - il s'agit donc d'une analyse à visée militante.

49 Au cours des mois de mars et avril 2011, dans les semaines précédant le lancement du débat sur la laïcité, le ministre de l'Intérieur Claude Guéant a fait un certain nombre de déclarations qui ont été considérées par certains commentateurs et acteurs de la vie politique française comme discriminatoires à l'égard des musulmans - ce dont Guéant s'est bien sûr défendu. Les quatre déclarations les plus commentées ont été les suivantes :

1) 17 mars 2011 : «Les Français, à force d'immigration incontrôlée, ont parfois le sentiment de ne plus être chez eux » (Déclaration sur Europe 1)

2) 21 mars 2011, à propos de la Libye : «Le président a pris la tête de la croisade pour mobiliser le Conseil de sécurité de l'Onu, la Ligue arabe et l'Union africaine » 3) 24 mars 2011 : Claude Guéant estime que les agents de service public «ne doivent pas porter de signes religieux, manifester une quelconque préférence religieuse, tout comme les usagers "

4) 4 avril 2011 : « En 1905, l'année de la loi sur la séparation des Eglises et de l'Etat, il $\mathrm{y}$ avait très peu de musulmans en France. Aujourd'hui, on estime qu'il y a à peu près 5 ou 6 millions de musulmans en France. C'est vrai que l'accroissement du nombre des fidèles de cette religion, un certain nombre de comportements, posent problème "

Sur la déclaration (1), j'ai choisi de m'intéresser à deux phénomènes linguistiques :

- l'utilisation de l'article défini "les", dans "les Français", qui suggère que les individus incommodés par les musulmans représentent en réalité tous les Français (et qui suggère par ailleurs que les musulmans ne sont pas français) ;

- l'utilisation de l'expression figurée " chez eux ", dans l'expression " ne se sentent plus chez eux ». Cette métaphore suggère que le système qui gouverne la sphère domestique privée vaut aussi pour la sphère politique à l'échelle nationale (par exemple, quelqu'un qui pénétrerait dans votre domaine privé sans y avoir été invité pourrait être à bon droit considéré comme un intrus, et chassé sans autre forme de procès; on voit ce que donne cette règle appliquée à l'immigration).

51 Sur la déclaration (2) : Guéant a été massivement critiqué pour avoir utilisé un terme religieux afin de désigner l'action de Nicolas Sarkozy, et pour avoir ainsi proposé un cadrage de l'intervention militaire en Libye comme une offensive religieuse du monde occidental contre une nation musulmane. Guéant s'est défendu en alléguant du second sens, non religieux, du mot "croisade», attesté dans les dictionnaires français. La conception du sens qui sous-tend la justification de Guéant ne correspond pas à la réalité des processus interprétatifs. Elle présuppose que l'interprétation des discours se ferait de façon totalement décontextualisée, sans interférence aucune de ce que le 
destinataire du discours sait des préférences idéologiques du locuteur à l'origine de la déclaration, et des enjeux qui caractérisent le contexte politique dans lequel les propos ont été tenus. Par ailleurs, la justification de Guéant suppose que les différents sens d'un même mot seraient complètement étanches les uns aux autres, et qu'il serait possible d'en activer un à l'exclusion de tous les autres. En bref, la justification de Guéant peut être vue de deux façons: soit elle témoigne d'une conception incroyablement naïve du fonctionnement de la signification des mots, soit elle reflète une forme de malhonnêteté intellectuelle.

Sur la déclaration (3), Guéant recourt à ce que Perelman appellerait un argument quasilogique - plus précisément, quelque chose comme un argument de réciprocité : si on demande aux agents du service public de ne pas porter de symboles religieux ou de ne pas manifester leur préférence religieuse, alors, les usagers du service public doivent faire de même. A l'examen, la symétrie apparemment indiscutable de la formule ne tient pas: l'exigence de neutralité religieuse des agents du service public est justifiée par le fait que ces agents, d'une certaine façon, représentent l'Etat dans l'exercice de leur profession. Ce n'est absolument pas le cas des usagers, qui, dans leur recours au service public, ne représentent qu'eux-mêmes, et n'ont donc pas à respecter l'exigence de neutralité.

Enfin, la déclaration (4) appelle quatre commentaires. En premier lieu, on soulignera les variations dans la formulation de l'importance de la population musulmane en France, qui ne facilite pas les comparaisons : Guéant affirme ainsi qu'elle est passée de "très peu " à " cinq à six millions ". Par ailleurs, cette assertion fait peu de cas de l'importance de la sphère musulmane (et en particulier de l'Afrique du Nord) dans ce qui constituait l'Empire français en 1905. L'expression " c'est vrai que ", quant à elle, marque la concession en français, et invite l'analyste à s'interroger sur la part de l'électorat à laquelle Guéant «concède » que le nombre croissant de musulmans pose problème (on peut ainsi penser que Guéant vise, par son discours, l'électorat d'extrême droite). Enfin, l'évocation non-spécifiée de "comportements» qui "poseraient problème», et leur attribution implicite aux musulmans, nourrit un discours accusatoire irréfutable dirigé contre la communauté musulmane (irréfutable non pas parce qu'il serait vrai, mais parce qu'il est insuffisamment spécifié pour faire l'objet d'une réfutation en règle).

La conclusion de cette analyse est que les déclarations de Guéant sont clairement discriminatoires, en ce qu'elles proposent une représentation de la société française comme étant le théâtre d'une confrontation conflictuelle entre les musulmans d'une part, et « les Français » de l'autre.

\section{Caractérisation de l'étude E / de l'étude M}

55 Sur la base de ces deux études de cas de discours politiques, je vais à présent chercher à dégager quelques caractéristiques permettant de distinguer les approches dont elles relèvent (épistémique versus militante).

Commençons par les plus triviales:

- Supports de diffusion : ces deux études ont été produites pour, et diffusées par, des médias différents : toutes deux peuvent être trouvées sur l'Internet, mais l'étude $\mathrm{M}$ a été publiée dans la rubrique "tribune» du site de mon laboratoire de recherche (http://www.lcp.cnrs.fr/spip.php ?article133), alors que l'étude E a été publiée dans 
une revue scientifique en ligne (revue Synergies Italie, numéro spécial « Euphémismes et stratégies d'atténuation du dire »).

- Destinataires visés : cette différence de support reflète et confirme le fait que chacune de ces études vise une classe de destinataires différente. L'étude E s'adresse prioritairement à des pairs issus de la communauté académique, alors que l'étude $\mathrm{M}$ du moins en théorie - s'adresse davantage au grand public (même s'il y a peu de chance que ledit grand public s'égare sur Internet et tombe sur le site d'un laboratoire de recherche).

- Degré de technicité : ces deux caractéristiques (support et audience visée) rendent compte du fait que l'étude $\mathrm{E}$ est beaucoup plus technique que l'étude $\mathrm{M}$. Elle utilise plus de termes spécialisés, elle se réfère constamment à des articles ou ouvrages écrits par des pairs, etc. En revanche, la compréhension de l'étude $M$ ne nécessite aucune expertise spécifique dans le champ de l'analyse du discours ou des théories de l'argumentation - bien que le recours aux marqueurs discursifs de la vulgarisation scientifique qu'on y trouve ( $\mathrm{X}$, ce qu'on appelle $\mathrm{Y} »$, « $\mathrm{X}$, ce qui veut dire $\mathrm{Y} » .$. ) rappelle au lecteur que l'analyse a été originellement élaborée sur la base d'une expertise académique.

- Genre discursif: ces observations amènent à conclure que les études $\mathrm{M}$ et $\mathrm{E}$ n'appartiennent simplement pas à un même genre discursif. Alors que $(E)$ obéit dans l'ensemble aux contraintes d'un article scientifique, (M) relève du discours polémique voire du discours pamphlétaire, tel que le caractérise Marc Angenot (1982) par exemple. Cette caractérisation générique divergente des deux études permet de rendre compte d'autres caractéristiques de ces textes ; en particulier :

- La différence de longueur entre les deux textes. Cette longueur est contrainte pour l'étude (E) (autour de 5600 mots), libre pour l'étude (M) (autour de 2500 mots $^{18}$ ).

- Des différences stylistiques et énonciatives. (E) présente les traits classiques de l'écriture scientifique : masquage massif de l'auteur, formes passives, nominalisations, etc. En revanche, l'auteur de $\mathrm{M}$ est fortement présent, à travers l'utilisation de « je », et plus généralement, à travers un style plus "flamboyant ». A ce propos, j'ajouterai un commentaire qui n'est en rien technique, mais qui me parait cependant pertinent (quoique pas très glorieux): l'écriture d'un texte polémique procure un plaisir très différent de celui que l'on peut ressentir lors de la rédaction d'un article scientifique. Il me semble que ce plaisir a quelque chose à voir avec un fantasme de puissance, avec le rêve de pouvoir, par la force des mots, à distance et sans grand risque, mettre K.-O. un adversaire. Or, écrit Bourdieu, «Le plaisir de se sentir malin, démystifié et démystificateur, de jouer les désenchantés désenchanteurs, est au principe de beaucoup d'erreurs scientifiques $»^{19}$.

Les deux dernières différences que je voudrais souligner sont sans doute les plus importantes.

- D'abord, les études $\mathrm{M}$ et $\mathrm{E}$ poursuivent des buts de nature très différente, et cherchent à répondre à des questions distinctes. $(\mathrm{M})$ «Les déclarations de Claude Guéant sontelles discriminatoires à l'égard de la communauté musulmane?» (ce qui est une question politique); (E): "Quel rôle joue l'expression de l'accord dans un genre interactionnel souvent caractérisé comme centré sur le désaccord (un débat politique)?» (ce qui est une question théorique, le débat Bayrou/Royal étant un prétexte à son exploration). Le statut de la réponse à cette question diffère également de (E) à (M). Je dois avouer que, dans une certaine mesure, je connaissais déjà la conclusion à laquelle j'arriverais lorsque j'ai initié l'étude M (il n'est pas nécessaire de 
disposer d'une intuition politique surdéveloppée pour pressentir que le discours de Guéant a des chances d'être discriminatoire, même si les procédés discursifs portant ce caractère discriminatoire ne sont pas prédictibles par avance). En revanche, au départ de l'étude $\mathrm{E}, \mathrm{j}$ 'ignorais complètement où j'allais arriver en tirant le fil de l'expression de l'accord dans un débat.

- La dernière différence que je voudrais souligner a à voir avec le choix et la délimitation des données qui constituent le corpus de travail. Dans l'étude E, l'objet soumis à l'investigation est " bi-face ", en ce sens qu'il intègre deux discours opposés, le pour et le contre, le discours et le contre-discours. Le choix d'un tel objet invite l'analyste à adopter une approche symétrique du discours de Bayrou et du discours de Royal, ce qui l'amène à trouver assez naturellement une position d'extériorité par rapport à son objet et au questionnement qu'il mène. La situation est très différente pour l'étude M. Les analyses portent exclusivement sur le discours de Guéant ; certains procédés discursifs y sont identifiés et interprétés comme discriminatoires ; mais je me garderais bien d'affirmer qu'ils sont spécifiques au discours de Guéant (on trouverait sans doute des choses bien surprenantes si on s'attelait à l'analyse d'un discours « de gauche » sur l'immigration). Et je dois avouer que j'ai eu le sentiment d'être un peu malhonnête en montrant par exemple comment l'utilisation de l'article défini "les » dans « les Français » contribuait à créer une vision de la société française comme une confrontation de deux blocs : il est plus que probable qu'un tel usage de «les » n'est pas propre à Guéant.

En bref, il m'a semblé, à travers cette expérience, qu'adopter une perspective militante supposait une part de malhonnêteté ou du moins de mauvaise foi, qui était peut-être la contrepartie d'une prétention à l'efficacité polémique ${ }^{20}$.

Je voudrais enfin conclure sur l'inconfort que génère pour moi l'élaboration parallèle de ces deux types d'analyse ${ }^{21}$. Cet inconfort est lié au fait que la frontière est parfois ténue entre eux, en raison du fait que l'un comme l'autre, d'une certaine façon, résultent de l'exercice d'une expertise (dans l'analyse des données aussi bien que dans l'écriture assurant sa restitution) ; pourtant, les enjeux y sont clairement distincts. Ils engagent leur auteur différemment : même si l'étude $\mathrm{M}$ nécessite, comme l'étude $\mathrm{E}$, une forme d'expertise, elle ne s'y limite pas (l'expertise est mise au service d'un objectif polémique). Il y a donc quelque chose comme une forme de malhonnêteté à mentionner mon statut (dans l'en-tête du document; il est aussi récupérable via la rubrique «membres » du site du laboratoire sur lequel le texte est publié), alors que ce n'est pas la raison de ma prise de parole. Pourtant, un effacement du statut, voire l'utilisation d'un pseudonyme, ne résoudraient pas le problème, l'écriture restant assez clairement rattachée à des pratiques professionnelles identifiables.

Enfin, dans l'étude $\mathrm{M}$, je soumets les données que j'examine à un questionnement critique très similaire tant aux pratiques évaluatives ordinaires qu'aux analyses normatives savantes - ce qui est clairement contradictoire avec le positionnement qui est le mien pour l'essentiel de mes recherches académiques.

61 Le passage d'un registre à l'autre n'est pas, on l'a dit, sans générer un inconfort certain. On y gagne en revanche par la diversité des visées communicatives qu'il permet de poursuivre ; peut-être est-il possible de "tenir » une diversification des productions expertes et des registres associés, d'un écrit académique et symétrique à un écrit militant engagé, en passant par des présentations orales donnant voix à «l'être-aumonde» derrière le chercheur; une condition en est à coup sûr une lucidité du 
chercheur sur son propre travail, et une transparence exigeante sur le statut de ses productions vis-à-vis de leurs destinataires, afin d'« éviter ces deux écueils opposés que sont soit l'abus de pouvoir (utiliser une compétence scientifique pour faire passer des convictions politiques), soit l'irresponsabilité (se désintéresser des usages normatifs qui peuvent être faits de ses analyses)» (Heinich 2002 : 119). A ces exigences de lucidité et de transparence peuvent s'associer les principes avancés par Neveu (2003: 112): "n'intervenir dans l'espace public que sur des questions où l'on a développé des recherches abouties, le faire avec le souci de réduire au maximum les coûts intellectuels des simplifications pédagogiques » - le privilège d'être payé par l'Etat pour faire de la recherche se traduisant par un devoir de faire profiter l'ensemble du corps social des acquis de ces recherches.

Ce problème du positionnement du chercheur s'est posé plus tôt, et plus frontalement, dans des disciplines comme la sociologie ou les sciences politiques, et les chercheurs qui y ont été confrontés ont élaboré une réflexion plus construite sur «les usages et l'utilité des sciences sociales dans le débat et l'action politique» (Neveu 2003:110); j'espère cependant avoir contribué à spécifier la façon dont la question pouvait se traduire dans le domaine de l'analyse argumentative, et à éclairer, par quelques remarques contrastives, certains traits discursifs caractéristiques de productions expertes distinctes, qui «rejouent» la fracture entre le savant et le politique. En ce sens, je ne fais que répéter la position qualifiée de dominante, et déplorée à ce titre, par Koren (2003 : 272), position selon laquelle «la seule option scientifique [c'est moi qui souligne] valide est la non-intervention »; non que l'intervention soit illégitime, mais elle relève soit de l'expertise, soit du militantisme, et constitue un " second temps » par rapport à l'attitude épistémique et à la visée de neutralité propres au travail de recherche.

\section{BIBLIOGRAPHIE}

\section{Référence des deux études citées}

Références Etude E : http://ressources-cla.univ-fcomte.fr/gerflint/Italie-special/

italiespecial.html

Références Etude M : http://www.lcp.cnrs.fr/spip.php ?article133

Références

Angenot, Marc. 1982. La parole pamphlétaire (Paris : Payot)

Barthe, Cyril \& Cyril Lemieux. 2002. «Quelle critique après Bourdieu? », Mouvements, 24-5, 33-38

Broch, Henri. 1989. Le Paranormal - ses documents, ses hommes, ses méthodes (Paris : Seuil)

Broch, Henri. 1991. Au Coeur de l'extra-ordinaire (Bordeaux : L'Horizon chimérique)

Cuniot, Alain. 1989. Incroyable mais... faux ! (Bordeaux : L'Horizon chimérique) 
Doury, Marianne. 2004. «La position de l'analyste de l'argumentation », Semen 17

(«Argumentation et prise de position : pratiques discursives », Amossy Ruth \& Roselyne Koren, éds), $149-163$

Doury, Marianne. 2008 : “"Ce n'est pas un argument !” Sur quelques aspects des théorisations spontanées de l'argumentation », Pratiques 139 (« Les Linguistiques populaires, Paveau, MarieAnne. \& Guy Achard-Bayle, éds), 111-128

Doury, Marianne \& Catherine Kerbrat-Orecchioni. 2011. « La place de l'accord dans l'argumentation polémique : le cas du débat Sarkozy/Royal (2007) », revue en ligne A contrario, $\mathrm{n}^{\circ}$ 16, 2011-2 (http://www.cairn.info/revue-a-contrario-2011-2.htm)

Eemeren, Frans H. van \& Bert Meuffels. 2002. « Ordinary arguers judgments on ad hominem fallacies », Eemeren, Frans H. van (éd.). Advances in Pragma-Dialectics (Amsterdam: SicSat / Newport News, Virginia: Vale Press), 45-64

Garssen, Bart. 2002. « Understanding argument schemes », Eemeren, Frans H. van (éd.). Advances in Pragma-Dialectics (Amsterdam: SicSat / Newport News, Virginia: Vale Press), 93-104

Gilbert, Michel A. 1997. Coalescent argumentation (Mahwah, New Jersey: Erlbaum)

Goodwin, Jean. 2012. « What is 'Responsible Advocacy' in Science? Good Advice », Goodwin. Jean. (éd.) Between Scientists and Citizens: Proceedings of a Conference at Iowa State University, June 1-2 (Ames, IA: Great Plains Society for the Study of Argumentation), 151-161

Govier, Trudy. 1987. Problems in Argument Analysis and Evaluation (Dordrecht / Providence: Foris Publications)

Govier, Trudy. 2001. A practical study of argument (5th edition) (Belmont: Wadsworth)

Heinich, Nathalie. 2002. « Pour une neutralité engagée », Questions de communication 2, 117-127

Heinich, Nathalie. 2004. « Pour en finir avec l'engagement des intellectuels », Questions de communication $5,149-160$

Jackson, Sally. 1988. « What can argumentative practice tell us about argumentation norms? ", Maier, Robert (éd.). Norms in argumentation. Proceedings of the Conference on Norms 1988 (Dordrecht / Providence: Foris Publications), 113-122

Koren, Roselyne. 2003. «L'engagement de l'un dans le regard de l'autre. Point de vue d'une linguiste », Questions de Communication 4, 271-277

Neveu, Erik. 2003. « Recherche et engagement : actualité d'une discussion », Questions de communication $3,109-120$

Lahire, Bernard. 2002 : « Objectivation sociologique, critique sociale et disqualification », Mouvements, 24-5, 46-52.

Plantin, Christian. 1998. « Les raisons des émotions », Bondi, M. (éd.), Forms of argumentative discourse / Per un'analisi linguistica dell'argomentare (Bologne : CLEUB), 3-50

Sitri, Frédérique. 2003. L'objet du débat. La construction des objets de discours dans des situations argumentatives orales (Paris: Presses de la Sorbonne Nouvelle)

Tavernier, Aurélie. 2012. « Une lecture rhétorique de l'expertise. La construction de l'ethos du sociologue dans les médias ", Léglise, Isabelle \& Nathalie Garric (éds), Discours d'experts et d'expertise (Berne : Peter Lang), 19-46 


\section{NOTES}

1. On se contentera ici de renvoyer aux dossiers consacrés à la question de l'engagement du chercheur par la revue Questions de Communication ( ${ }^{\circ} 2,3,4$ et 5, parus entre 2002 et 2004), et, plus spécifiquement, à la charge critique de la sociologie (Mouvements 24-5, 2002) ; ces dossiers reviennent sur l'émergence déjà ancienne de la question, et sur sa résurgence actuelle.

2. La solution adoptée est d'une cohérence toute relative, le premier ouvrage ayant reçu le statut de « corpus ", et les deux autres, de références théoriques...

3. http://www.lexpress.fr/actualite/environnement/nicolas-hulot-la-decroissance-n-est-pasun-gros-mot_507449.html

4. http://www.dailymotion.com/video/x5jzig_segolene-royal-dimanche-25-mai-2008_news, message de jeremove, fin mai 2008.

5. $\mathrm{AMHA}=$ « à mon humble avis »

6. Fr.sci.astrophysique, message de Benoit.d, 4 mai 2008.

7. Fr.soc.politique, message de Declairvaux33, 28 juillet 2008.

8. André Bernand, Pan du désert, 1977, Brill. P.XXIII.

9. http://www.digital-broadcast-channel.com/forums/index.php?showtopic=2118\&st=36

10. Au sens de «prise de position dans l'espace public », Heinich $2004: 154$.

11. Cette approche descriptive de la critique argumentative rejoint les courants de recherche qualifiés de pragmatiques évoqués par Barthe et Lemieux (2002) comme alternatives à la vision bourdieusienne du rôle critique de la sociologie. Ces courants pragmatiques prêtent attention aux compétences critiques des acteurs sociaux et aux capacités réflexives dont ils sont dotés, et les auteurs montrent que l'abandon, par le sociologue, d'une position de surplomb pour «cheminer auprès des acteurs" ne revient pas à renoncer à toute portée politique de la recherche.

12. Même si, pour Heinich, seul le registre descriptif « est spécifiquement celui du chercheur, alors que les registres évaluatif et prescriptif relèvent d'une normativité qui est du ressort soit de l'expert, soit du penseur, selon le type et le degré de spécialisation de la compétence » (2002: 119).

13. S'appuyant sur sa propre expérience, parfois douloureuse, des médias, Neveu rappelle ainsi que dans un tel contexte, « un savant, cela sert à dire qui a gagné » $(2003: 115)$.

14. On trouvera, dans Goodwin 2012, une présentation des termes dans lesquels se pose la question de l'intervention des scientifiques sur des sujets de politique publique, et une synthèse éclairante des principaux arguments pour et contre une telle intervention dans les domaines de la biologie liés aux ressources naturelles - cette synthèse fait bien sûr sens plus généralement pour tout ce qui relève d'une " policy advocacy ".

15. Il me semble que Lahire (2002) pointe un processus similaire lorsqu'il dénonce le glissement, d'une position revendiquée d'objectivation critique, à une disqualification masquée.

16. Catherine Kerbrat-Orecchioni et moi-même avons repris et approfondi cette problématique de l'expression de l'accord dans un débat télévisé dans un travail portant cette fois sur le débat opposant Nicolas Sarkozy et Ségolène Royal au cours de cette même campagne présidentielle (Doury et Kerbrat-Orecchioni, 2011).

17. Ou plutôt, qu'il l'est factuellement, mais pas rhétoriquement.

18. Mais ce n'est pas une propriété des textes militants, dont la longueur dépend du support de diffusion, et des contraintes qui lui sont attachées.

19. P. Bourdieu, Raisons pratiques. Sur la théorie de l'action; cité en exergue de Lahire 2002.

20. Efficacité dont il faut bien admettre le caractère tout relatif, aucun de mes deux textes « militants » n'ayant fait trembler sur ses bases le gouvernement Sarkozy.

21. J'ai été tentée de parler non pas d'inconfort, mais, plus radicalement, de schizophrénie, liée à cette double pratique de l'expertise; mais des discussions avec Christian Plantin m'ont 
convaincue qu'il n'y a rien de schizophrénique à adapter ma production à des situations diverses et aux enjeux qui les gouvernent; ce qui serait problématique, ce serait l'homogénéisation à toute force d'un footing (au sens de Goffman) appliqué indifféremment à toutes les circonstances de la vie - ce qui supposerait la subordination de la production académique à un engagement militant, ou, à l'inverse (et pire encore) l'envahissement du registre privé par une attitude académique distanciée interdisant toute participation active aux échanges argumentatifs qui animent notre quotidien.

\section{RÉSUMÉS}

Cet article propose d'aborder la question du positionnement du chercheur vis-à-vis de son objet (et en particulier, de la prise en charge d'une dimension critique), question qui traverse l'ensemble des sciences sociales, en la rapportant à l'expérience de l'auteure dans le champ des études en argumentation. Distinguant trois perspectives (positionnement descriptif, normatif, militant), elle met en parallèle deux analyses de discours politiques, obéissant à deux logiques différentes (épistémique pour l'une, politique pour l'autre), et en dégage quelques caractéristiques propres à ces logiques. Tout en reconnaissant la légitimité d'une préoccupation d'action critique, elle conclut sur la nécessité de reconnaître et entretenir la rupture entre le savant et le politique dans les études en argumentation.

This paper focuses on the issue of the researcher's attitude to his object (in particular, on the possibility of adopting a critical stance). This question concerns the whole realm of social sciences; here, it is tackled in the light of the author's experience in argumentation studies. She distinguishes three perspectives (descriptive positioning, normative positioning, militant positioning) and compares two analyses she produced of some political discourse. These analyses obey different logics (an epistemic and a political one), and display contrasting discursive and enunciative characteristics. While admitting that caring for critical activism is legitimate, the author concludes to the necessity of acknowledging and maintaining the split between what falls under science and what falls under politics in argumentation studies.

\section{INDEX}

Mots-clés : analyse du discours politique, critique, perspective descriptive, perspective militante, perspective normative

Keywords : critics, descriptive perspective, militant perspective, normative perspective, political discourse analysis

\section{AUTEUR}

\section{MARIANNE DOURY}

CNRS, Laboratoire Communication et Politique 